Canadian

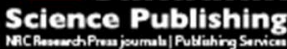

Applied Physiology, Nutrition, and Metabolism Physiologie appliquée, nutrition et métabolisme

\title{
Aspartame intake is associated with greater glucose intolerance in individuals with obesity
}

\begin{tabular}{|r|l|}
\hline Journal: & Applied Physiology, Nutrition, and Metabolism \\
\hline Manuscript ID & apnm-2015-0675.R2 \\
\hline Manuscript Type: & Brief communication \\
\hline Date Submitted by the Author: & 31 -Mar-2016 \\
\hline Complete List of Authors: & $\begin{array}{l}\text { Kuk, Jennifer; York University, } \\
\text { Brown, Ruth; York University, Kinesiology \& Health Science }\end{array}$ \\
\hline Keyword: & $\begin{array}{l}\text { body mass index < body composition, epidemiology, NHANES, nutrition } \\
\text { epidemiology < nutrition }\end{array}$ \\
\hline \multicolumn{2}{|l}{} \\
\hline
\end{tabular}

\section{SCHOLARONE \\ Manuscripts}


Aspartame intake is associated with greater glucose intolerance in individuals with obesity

Jennifer L Kuk, PhD and Ruth E Brown, PhD

School of Kinesiology and Health Science

York University, Toronto, Canada, M3J 1P3

Running Title: Sweeteners and Glucose Tolerance

\section{Address for Correspondence:}

Jennifer L. Kuk, PhD

School of Kinesiology and Health Science

York University

4300 Keele St., Toronto, ON, Canada

Toronto, Ontario, Canada, M3J 1P3

E-mail:jennkuk@yorku.ca

Phone: (416) 736-2100 ext. 20080

Fax: (416) 736-5772 


\begin{abstract}
This study examined whether sucrose, fructose, aspartame and saccharin influences the association between obesity and glucose tolerance in 2856 adults from the NHANES III survey. Aspartame intake significantly influenced the association between BMI and glucose tolerance (interaction: $\mathrm{P}=0.004)$, wherein only those reporting aspartame intake had a steeper positive association between BMI and glucose tolerance, than those reporting no aspartame intake. Therefore, consumption of aspartame is associated with greater obesity-related impairments in glucose tolerance.
\end{abstract}

Key Words: body mass index, epidemiology, National Health and Nutrition Examination Survey (NHANES) 


\section{Introduction}

Type 2 diabetes mellitus is a metabolic disorder characterized by glucose intolerance and insulin resistance leading to hyperglycemia (Kerner 2014). Obesity has been strongly linked with type 2 diabetes, and nearly $90 \%$ of individuals with diabetes have overweight or obesity (Saydah 2014). Sugar consumption is one of the common suspected culprits for the rise in obesity and may contribute to the development of type 2 diabetes (Hu 2010). Consequently, artificial sweeteners are commonly consumed by individuals with obesity and type 2 diabetes as they are thought to lower the caloric content and blood glucose response. However, in animal models, use of artificial sweeteners may also increase weight gain over time (Swithers 2013), and may promote glucose intolerance through the altered function and composition of intestinal microbiota (Suez 2014). In humans, there is very little evidence that artificial sweeteners promote glucose intolerance and there are only a few long-term studies on the use of sweeteners and weight change, and studies that report metabolic differences with intervention do not adjust for concurrent changes in body weight (Wiebe 2011). Further, there is no report on whether natural sugars or artificial sweeteners have an effect on diabetes risk independent of obesity.

Thus, the aim of this study is to determine whether natural sugars and artificial sweetener consumption modifies the association between glucose tolerance, insulin resistance, fasting glucose and BMI. 


\section{Methods}

Participants

The Third National Health and Nutrition Examination Survey (NHANES III) is a nationally representative cross-sectional survey conducted in the United States between 1988 and 1994 in 33,994 persons, aged 2 months and older. Participants were examined at home and at a mobile exam center for various markers of health, including demographics, medical history, dietary practices, blood profile and anthropometrics (CDC 1994; CDC 1996). Participants gave informed written consent, and the study protocol was approved by the National Center for Health Statistics.

Secondary analyses conducted for this study utilized 2856 adults with complete data for oral glucose tolerance, weight, height and diet, and who were not taking medications for diabetes. Age, sex, education, ethnicity (White, or non-White), and smoking status (never, current and former) were self-reported by questionnaire. Body mass index (BMI) was calculated using measured height and weight.

\section{Metabolic Measures}

The oral glucose tolerance test (OGTT) was conducted at the mobile examination center and was limited to a subset of individuals aged 40-74 years old without previously diagnosed type 2 diabetes and who were not taking insulin, after an overnight fast ( $>8$ hours). Blood was drawn from the antecubital vein by a trained phlebotomist according to a standardized protocol. Participants received a 75-g glucose-equivalent oral glucose challenge and a second blood sample was drawn 2 hours later. Fasting and 2-h plasma glucose levels were measured by a hexokinase enzymatic reference method (Roche Cobas Mira, Indianapolis, IN). Homeostatic 
model assessment of insulin resistance (HOMA-IR) was calculated by dividing the product of fasting plasma glucose $(\mathrm{mmol} / \mathrm{L})$ and insulin $(\mathrm{mU} / \mathrm{L})$ by 22.5 .

\section{Dietary Intake}

At the mobile examination center, two dietary interviewers administered the dietary questionnaire and recorded the types and amounts of all foods and beverages consumed by the participant in the last 24 hours. Data on specific sugar (sucrose and fructose) and artificial sweetener (aspartame and saccharin) intake was available from The Total Nutrient Intake File. Individuals were classified as consumers or non-consumers of artificial sweeteners (yes or no) and by high and low natural sugar intake (top $80 \%$ versus bottom $20 \%$ ).

\section{Statistical analysis}

Participant characteristics are presented as mean (SE) or frequencies. Differences in the relationship between glucose tolerance, fasting glucose and insulin resistance (outcomes) with BMI (continuous correlate) by sugar/sweetener intake (categorical) were assessed using elementwise regression (proc surveyreg which uses generalized least squares estimation) with examination of BMI by sugar/sweetener interaction and main effects with adjustment for potential confounders (age, sex, smoking status, education, and ethnicity - fixed effects). All statistical analyses were performed using SAS v9.4 survey procedures weighted to be representative of the U.S. population. Statistical significance was set at alpha $<0.05$. 


\section{Results}

Individuals consuming artificial sweeteners (aspartame or saccharin) had a subtly higher BMI (28 versus $27 \mathrm{~kg} / \mathrm{m}^{2}$ ), and were more likely to be female (Table 1, $\mathrm{P}<0.05$ ). Those consuming high sucrose, high fructose and no aspartame were significantly younger, while those consuming saccharin were significantly older than those who $\operatorname{did} \operatorname{not}(\mathrm{P}<0.05)$. There were no differences in glucose tolerance, HOMA-IR or fasting plasma glucose between those consuming high sugars or artificial sweeteners and those who did not.

The interaction and main effects of BMI and sugars and sweeteners are presented in Figure 1. Sucrose and fructose intake were not significantly related with glucose tolerance, HOMA-IR or fasting glucose independent of BMI $(\mathrm{P}>0.10)$. There was a significant aspartame by $\mathrm{BMI}$ interaction effect $(\mathrm{P}=0.004)$, wherein the slope of the association between $\mathrm{BMI}$ and glucose tolerance was steeper in those reporting aspartame intake (Figure 1). Similar observations were seen between aspartame, BMI and fasting glucose (not shown). Saccharin intake did not alter the association between BMI and glucose tolerance, fasting glucose or HOMA-IR $(\mathrm{P}>0.10)$. 


\section{Discussion}

The results of the current study illustrate that aspartame consumption is associated with augmented obesity-related deteriorations in glucose tolerance and fasting glucose. Thus, more research is needed to better understand the weight management benefits of artificial sweetener consumption over natural sugars against the potential increased diabetes risk, particularly for those with obesity.

Due to the potential health dangers and weight implications of natural sugars, use of lowcalorie sweeteners has grown in popularity (Sylvetsky 2012), and many newer artificial sweeteners are now consumed beyond those examined here (Mayo Clinic 2016). Use of lowcalorie sweetener substitutes are reported to be associated with clear reductions in caloric intake and modest reductions in body weight (Miller 2014). However, there is surprisingly little research showing a long term health benefit of low-caloric sweeteners (Wiebe 2011; Miller and Perez 2014), particularly when changes in body weight are considered (Wiebe 2011). This study provides evidence that consumption of aspartame may be associated with greater diabetes risk in individuals with higher adiposity. Aspartame is reported to be associated with changes in gut microbiota that are associated with impairments in insulin resistance in lean (Suez 2014) and obese rodents (Palmnäs 2014). We observe that aspartame was related with significantly greater impairments in glucose tolerance for individuals with obesity, but not lean individuals. In fact, our results suggest a beneficial effect of aspartame in lean individuals. However, as there are very few lean individuals in the population who reported consuming aspartame, this requires further investigation.

From mice models, there is also evidence that saccharin consumption induce changes in intestinal microbiota that result in the development of glucose intolerance (Suez 2014). In the 
current study, there was a non-significant $0.45 \mathrm{mM}$ higher 2 -hour glucose in those who consumed saccharin versus those who did not. However, this difference may be clinically relevant as individuals with obesity who consumed saccharin had a mean impaired glucose tolerance versus a normal glucose tolerance for those with obesity who did not consume saccharin, suggesting that we may have been underpowered for this analysis. Suez et al. (2014) demonstrate that 7 days of saccharin consumption is associated with impairments in glucose tolerance in lean individuals who do not habitually consume non-caloric sweeteners. Thus, there is evidence from both rodent and human research suggesting that saccharin may be associated with deleterious health effects.

In general, the evidence suggesting a metabolic effect of artificial sweeteners in humans is controversial (Wiebe 2011; Swithers 2013). Epidemiological evidence suggests that diet soda consumption is associated with a higher risk for developing type 2 diabetes, though this observation is not consistently observed (Hu and Malik 2010). Further, it is unclear if the increased diabetes risk associated with diet soda consumption remains independent of differences in adiposity (O'Connor 2015). Interestingly, a meta-analysis suggesting minimal health effects of sweeteners, also report that most examinations have been in non-obese populations (Wiebe 2011). Together, these results may indicate that certain artificial sweeteners such as aspartame have negative effects on glucose tolerance in individuals with obesity who are at the greatest risk for developing type 2 diabetes.

Consumption of sucrose is suspected to be a culprit in the rise in obesity prevalence $(\mathrm{Hu}$ and Malik 2010). Chronic consumption of high sugar diets are associated with increased inflammation, glucose intolerance and greater weight gain in rats (Sweazea 2010). In humans, consumption of sugar sweetened beverages are associated with higher caloric intake, greater 
obesity and greater risk for type 2 diabetes (Hu and Malik 2010). However, many reports demonstrating a negative effect of sugar intake on diabetes risk are confounded by the effects of obesity and greater weight gain. Further, some suggest that the diabetes risk associated with sugar may be less than what is observed for artificially sweetened sodas (Sakurai 2014). In the current study, we do not observe an independent effect of sucrose consumption on glucose tolerance independent of BMI, supporting the notion that it is calories as opposed to sugar that may be leading to obesity and increased diabetes risk (Hu and Malik 2010).

In humans, fructose consumption is thought to be related with increased visceral adiposity and dyslipidemia even without weight gain (Maersk 2012). Although visceral adiposity and dyslipidemia are both associated with glucose intolerance (Borel 2014; Boden 2002), we did not observe that high fructose consumption has an independent effect on glucose tolerance. This may indicate that our cut-off used to denote 'high' fructose consumption may not have been sufficiently high, or that the effects of fructose on visceral adiposity were not sufficiently large to have a negative impact on glucose tolerance independent of BMI.

The results of this study are based on 24-hour self-reported diet and may not be reflective of chronic intake patterns. However, it is unclear whether chronic ingestion is needed to result in changes in glucose tolerance, or if these results also apply to the newer artificial sweeteners that are now currently used. Further, we are unsure if there is a dose-response relationship between sweeteners and changes in glucose tolerance, and the cross-sectional results observed here may be subject to reverse causation. Finally, due to the multiple associations examined, there is the possibility that the observations here are due to chance. Nevertheless, these results are weighted to be representative of the U.S population and illustrate on a population level that sweetener 
consumption may have clinically relevant impacts on glucose tolerance and type 2 diabetes risk independent of BMI.

In conclusion, aspartame artificial sweetener consumptions may be associated with greater glucose intolerance, particularly for those with obesity. Future research is needed to determine whether from an obesity and diabetes perspective, it may be prudent to limit all sweetener consumption.

\section{Conflict of Interest}

The authors have no relevant conflicts of interest to disclose. 


\section{References}

1994. Centers for Disease Control and Prevention (CDC). National Center for Health

Statistics (NCHS). Plan and Operation of the Third National Health and Nutrition

Examination Survey, 1988-94. . Hyattsville, MD: U.S. Dept. of Health and Human Services, Public Health Service, Centers for Disease Control and Prevention, National Center for Health Statistics.

1996. Centers for Disease and Prevention (CDC). National Center for Health Statistics (NCHS). Analytic and Reporting Guidelines for the Third National Health and Nutrition Examination Survey, NHANES III (1988-94). . Hyattsville, MD: Department of Health and Human Services, Centers for Disease Control and Prevention.

Boden, G. 2002. Interaction between free fatty acids and glucose metabolism. Curr Opin Clin Nutr Metab Care 5(5): 545-549.

Borel, A.L., Nazare, J.A., Smith, J., Aschner, P., Barter, P., Van Gaal, L., et al. 2014. Visceral, subcutaneous abdominal adiposity and liver fat content distribution in normal glucose tolerance, impaired fasting glucose and/or impaired glucose tolerance. Int J Obes (Lond).

Hu, F.B. and Malik, V.S. 2010. Sugar-sweetened beverages and risk of obesity and type 2 diabetes: Epidemiologic evidence. Physiology \& behavior 100: 47-54.

Kerner, W. and Bruckel, J. 2014. Definition, classification and diagnosis of diabetes mellitus. Exp Clin Endocrinol Diabetes 122: 384-6.

Maersk, M., Belza, A., Stødkilde-Jørgensen, H., Ringgaard, S., Chabanova, E., Thomsen, H., et al. 2012. Sucrose-sweetened beverages increase fat storage in the liver, muscle, and visceral fat 
depot: a 6-mo randomized intervention study. The American Journal of Clinical Nutrition 95: 283-289.

Mayo Clinic. 2016. Artificial sweeteners and other sugar substitutes [online]. Available from http://www.mayoclinic.org/healthy-lifestyle/nutrition-and-healthy-eating/in-depth/artificialsweeteners/art-20046936 [accessed 4 March 2016].

Miller, P.E. and Perez, V. 2014. Low-calorie sweeteners and body weight and composition: a meta-analysis of randomized controlled trials and prospective cohort studies. Am J Clin Nutr 100: 765-77.

O'Connor, L., Imamura, F., Lentjes, M. A., Khaw, K. T., Wareham, N. J. and Forouhi, N. G. 2015. Prospective associations and population impact of sweet beverage intake and type 2 diabetes, and effects of substitutions with alternative beverages. Diabetologia 58(7): 1474-1483.

Palmnäs, M. S. A., Cowan, T. E., Bomhof, M. R., Su, J., Reimer, R. A., Vogel, H. J., et al. 2014. Low-Dose Aspartame Consumption Differentially Affects Gut Microbiota-Host Metabolic Interactions in the Diet-Induced Obese Rat. PLoS ONE 9(10): e109841.

Sakurai, M., Nakamura, K., Miura, K., Takamura, T., Yoshita, K., Nagasawa, S. Y., et al. 2014. Sugar-sweetened beverage and diet soda consumption and the 7-year risk for type 2 diabetes mellitus in middle-aged Japanese men. Eur J Nutr 53(1): 251-258.

Saydah, S., Bullard, K.M., Cheng, Y., Ali, M.K., Gregg, E.W., Geiss, L., et al. 2014. Trends in cardiovascular disease risk factors by obesity level in adults in the United States, NHANES 1999-2010. Obesity 22: 1888-1895. 
Suez, J., Korem, T., Zeevi, D., Zilberman-Schapira, G., Thaiss, C.A., Maza, O., et al. 2014. Artificial sweeteners induce glucose intolerance by altering the gut microbiota. Nature 514: 181186.

Sweazea, K.L., Lekic, M., and Walker, B.R. 2010. Comparison of mechanisms involved in impaired vascular reactivity between high sucrose and high fat diets in rats. Nutrition \& Metabolism 7: 48-48.

Swithers, S.E., Sample, C.H., and Davidson, T.L. 2013. Adverse effects of high-intensity sweeteners on energy intake and weight control in male and obesity-prone female rats.

Behavioral neuroscience 127: 262-274.

Sylvetsky, A. C., Welsh, J. A., Brown, R. J. and Vos, M. B. 2012. Low-calorie sweetener consumption is increasing in the United States. The American Journal of Clinical Nutrition 96(3): 640-646.

Walker, R. W., Dumke, K. A. and Goran, M. I. 2015 Fructose content in popular beverages made with and without high-fructose corn syrup. Nutrition 30(7): 928-935.

Wiebe, N., Padwal, R., Field, C., Marks, S., Jacobs, R., and Tonelli, M. 2011. A systematic review on the effect of sweeteners on glycemic response and clinically relevant outcomes. BMC Medicine 9: 123-123. 
Table 1. Characteristics of adults from NHANES III (1988-1994) according to sucrose, aspartame and saccharin intake

\begin{tabular}{|c|c|c|c|c|c|c|c|c|}
\hline & $\begin{array}{c}\text { Low } \\
\text { Sucrose }\end{array}$ & $\begin{array}{c}\text { High } \\
\text { Sucrose }\end{array}$ & $\begin{array}{c}\text { Low } \\
\text { Fructose }\end{array}$ & $\begin{array}{c}\text { High } \\
\text { Fructose }\end{array}$ & $\begin{array}{c}\text { No } \\
\text { Aspartame }\end{array}$ & Aspartame & $\begin{array}{c}\text { No } \\
\text { Saccharin }\end{array}$ & Saccharin \\
\hline Males N (\%) & $1018(44.6)$ & $345(60.3) *$ & $1135(44.5)$ & $228(60.3) *$ & $1126(50.5)$ & $237(37.7)^{*}$ & $1238(49.0)$ & $125(38.1)^{*}$ \\
\hline BMI $\left(\mathrm{kg} / \mathrm{m}^{2}\right)$ & $27.5(0.2)$ & $27.0(0.3)$ & $27.3(0.2)$ & $27.7(0.7)$ & $27.1(0.2)$ & $28.2(0.3) *$ & $27.3(0.2)$ & $28.0(0.4)$ \\
\hline Normal Weight (\%) & $30.2[1.4]$ & $7.0[0.7]$ & $32.3[1.6]$ & $5.1[0.8]$ & $29.2[1.2]$ & $8.1[0.9]$ & $33.7[1.4]$ & $3.6[0.5]$ \\
\hline Overweight (\%) & $27.5[1.2]$ & $8.5[0.8]$ & $31.4[1.3]$ & $4.6[0.6]$ & $25.4[1.0]$ & $10.6[1.0]$ & $31.5[1.3]$ & $4.5[0.6]$ \\
\hline Fasting Glucose (mM) & $5.6(0.1)$ & $5.6(0.1)$ & $5.6(0.1)$ & $5.6(0.1)$ & $5.6(0.1)$ & $5.6(0.1)$ & $5.6(0.5)$ & $5.8(0.2)$ \\
\hline HOMA-IR & $3.0(0.1)$ & $2.6(0.1)$ & $2.9(0.1)$ & $3.1(0.4)$ & $2.8(0.1)$ & $3.0(0.3)$ & $2.9(0.1)$ & $3.1(0.4)$ \\
\hline Sucrose (gm) & $32(1)$ & $120(6)^{*}$ & $49(3)$ & $67(3)^{*}$ & $54(3)$ & $46(4)$ & $52(2)$ & $46(6)$ \\
\hline Fructose (gm) & $20.4(0.7)$ & $28.4(1.4) *$ & $16.2(0.3)$ & $62.0(2.5)^{*}$ & $24.2(0.8)$ & $17.0(0.6)^{*}$ & $22.8(0.7)$ & $17.4(1.3) *$ \\
\hline Aspartame (mg) & $73.5(5.3)$ & $57.9(11.8)$ & $77.2(5.1)$ & $21.9(5.7)^{*}$ & 0 & $\begin{array}{c}252.0 \\
(11.1)^{*}\end{array}$ & $65.8(4.9)$ & $99.8(15.4)^{*}$ \\
\hline
\end{tabular}

Values presented are mean (SE) or prevalence [SE].

* Significantly different from Low or No intake $(\mathrm{P}<0.05)$. 


\section{Figure Legends}

Figure 1. Association between 2-hour glucose and BMI according to low and high levels of sucrose (panel A), fructose (panel B), saccharin (panel C), and aspartame (panel D).

$\mathrm{BMI}=$ body mass index. Regression lines are adjusted for age, sex, smoking status education and ethnicity with standard deviation in estimates shown at the $5^{\text {th }}$ and $95^{\text {th }}$ percentile points. 

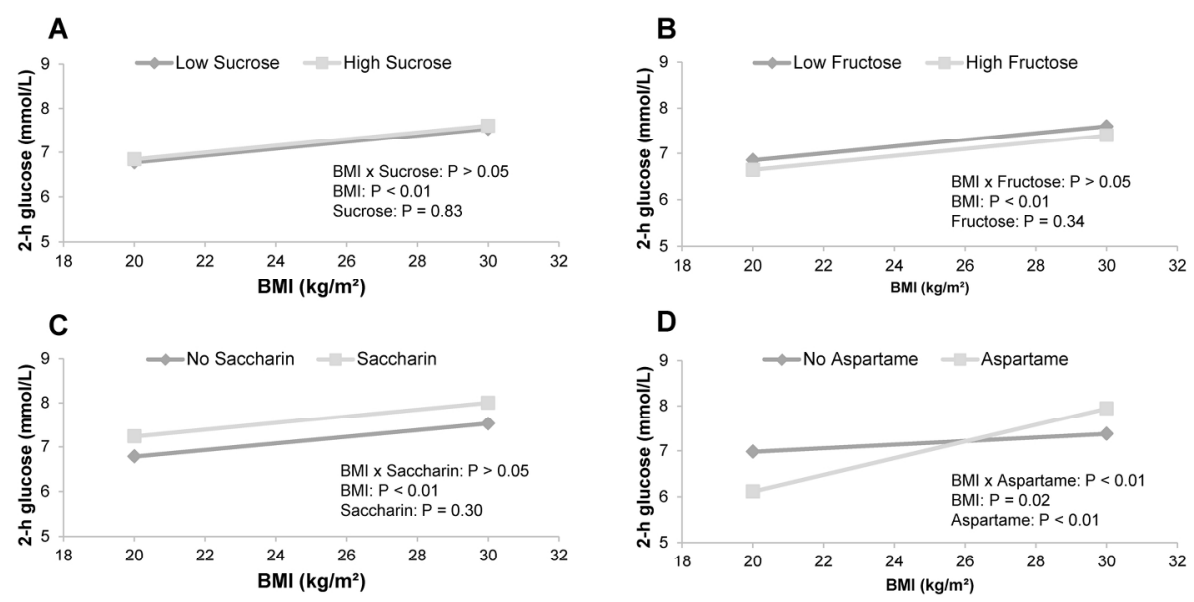

Figure 1. Association between 2-hour glucose and BMI according to low and high levels of sucrose (panel A), fructose (panel B), saccharin (panel C), and aspartame (panel D).

BMI = body mass index. Regression lines are adjusted for age, sex, smoking status education and ethnicity with standard deviation in estimates shown at the 5th and 95th percentile points $190 \times 142 \mathrm{~mm}(300 \times 300$ DPI $)$ 